http://dx.doi.org/10.35381/r.k.v6i11.1209

\title{
La familia en Ecuador: Un enfoque desde lo jurídico
}

The family in Ecuador: Has a legal approach

\author{
Nancy Susana Cárdenas-Yánez \\ susana.cardenas@ucuenca.edu.ec \\ Universidad de Cuenca, Cuenca \\ Ecuador \\ https://orcid.org/0000-0001-6743-2500 \\ Vicente Manuel Solano-Paucay \\ vichosolano@hotmail.com \\ Universidad de Cuenca, Cuenca \\ Ecuador \\ https://orcid.org/0000-0003-3955-8151 \\ Lourdes Álvarez-Coronel \\ lourdesalvarezcoronel@gmail.com \\ Universidad de Cuenca, Cuenca \\ Ecuador \\ https://orcid.org/0000-0001-9166-853X \\ María Elena Coello-Guerrero \\ maría.coello@ucuenca.edu.ec \\ Universidad de Cuenca, Cuenca \\ Ecuador \\ https://orcid.org/0000-0003-0181-0630
}

Recepción: 20 de enero 2021

Revisado: 15 de febrero 2021

Aprobación: 15 de abril 2021

Publicación: 01 de mayo 2021 


\title{
RESUMEN
}

En todos los lugares del mundo la familia constituye un pilar fundamental para el desarrollo social, económico, cultural, científico entre otros. Para el pueblo ecuatoriano desde lo jurídico ocupa un espacio especial. La investigación tiene como objetivo general analizar desde la perspectiva jurídica la visión de la familia en Ecuador. La misma se llevó a cabo desde el método analítico-sintético y se desarrolló desde un tipo descriptivo documental, empleando la observación y análisis de contenido jurídico con el propósito de descubrir las ideas primordiales de leyes construir un sumario teórico del caso de estudio, los mismos se presentan en los resultados investigativo. Se encontró disgregada la legislación concerniente con la familia en diversos cuerpos legales, se interpreta que ocasiona menoscabo en cuanto a la protección de las familias, es decir se debe recurrir a varias normas y leyes para la solución legal relacionado a los diferentes casos por ejemplo alimentación, violencia, entre otros.

Descriptores: Familia; derecho; bienestar social; sistema jurídico; parentesco. (Palabras tomadas del Tesauro UNESCO).

\begin{abstract}
In all parts of the world, the family constitutes a fundamental pillar for social, economic, cultural, scientific development, among others. For the Ecuadorian people, from the legal point of view, it occupies a special space. The general objective of the research is to analyze the vision of the family in Ecuador from a legal perspective. It was carried out from the analytical-synthetic method and was developed from a descriptive documentary type, using the observation and analysis of legal content with the purpose of discovering the main ideas of laws, constructing a theoretical summary of the case study, the same are presented in the research results. The legislation concerning the family was found disaggregated into various legal bodies, it is interpreted that it causes impairment in terms of the protection of families, that is, various norms and laws must be resorted to for the legal solution related to different cases, for example food, violence, among others.
\end{abstract}

Descriptors: Family; right; social welfare; legal system; relationship. (Words taken from UNESCO Thesaurus). 


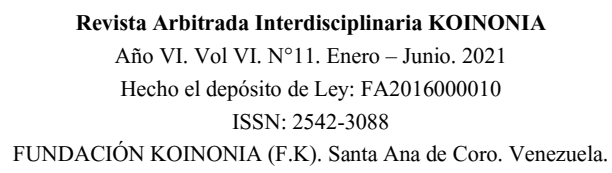

Nancy Susana Cárdenas-Yánez; Vicente Manuel Solano-Paucay; Lourdes Álvarez-Coronel; María Elena Coello-Guerrero

\section{INTRODUCCIÓN}

El presente análisis aborda a la institución de la familia desde el enfoque histórico de su surgimiento y evolución, su posterior conceptualización y su regulación jurídica en el ámbito ecuatoriano. La familia constituye el elemento esencial en el desarrollo del ser humano, representa la primera organización social subsistiendo hasta nuestros días, claro está con una serie de ajustes a las nuevas necesidades de las personas y las exigencias de una sociedad que se encuentra frente a nuevos retos, nuevas formas de comunicarse por la presencia cada vez más marcada de la tecnología.

Los diversos enfoques que han estudiado esta institución coinciden en que la misma requiere de un conjunto de normas que la regulen a fin de que cada uno de los engranajes que la componen funcione correctamente. Es apreciada como el grupo de individuos hermanadas ya sea por afinidad, unión sanguínea y afectividad, lo cual determina el surgimiento de una serie de derechos y obligaciones de carácter social o patrimonial, que se encuentran reguladas en el caso ecuatoriano en distintos cuerpos normativos como son: Código Civil, Código Orgánico de la Niñez y Adolescencia, Código del Trabajo, entre otras normas orgánicas o secundarias, cuyos objetivos es establecer los deberes y derechos de los miembros que conforman la familia, según los vínculos que nacen por ser parte de ellos y que en el presente artículo se referirán, con el fin de otorgar un panorama amplio sobre la importancia dentro del Estado de enmarcar y proteger a esta institución y sus diversas formas de manifestarse para lograr lo que constitucionalmente se proclama: Una convivencia ciudadana en diversidad y armonía con el fin de alcanzar el buen vivir, el sumak kawsay.

Dentro del género humano resulta evidente la necesidad constante de la búsqueda más allá de lo emocional o de la prolongación de la especie, de un vínculo más fuerte; esto es, de dar origen a lo que conocemos como familia. Puede afirmarse que la familia es apreciada como un conjunto de personas unidas por lazos consanguíneos, de afinidad como el matrimonio o la adopción, inclusive hay corrientes que consideran, además, que este vínculo puede nacer de lazos netamente afectivos, originados, por ejemplo por la 
migración, que a más de constituir un fenómeno social que ha generado recursos económicos, ha sido un fenómeno que dentro de la familia ha motivado cambios, percepciones, nuevos problemas, relaciones y conceptos, en donde los hijos de migrantes han debido ser criados, protegidos, cuidados, por personas que sin mantener vínculo de ninguna clase, lo han hecho como si fueran de su propia familia, desarrollando afectos entre ellos.

El autor Carbonell (2012) citado por Oliva \& Villa (2014, p.12) señala que "desde una concepción tradicional, se puede observar que "la familia ha sido el lugar primordial donde se comparten y gestionan los riesgos sociales de sus miembros". Por otro lado, de acuerdo a la autora Benítez (2017), la familia es una institución que se encuentra en todas las sociedades humanas y es reconocida entre los aspectos más valorados en la vida de los individuos. Constituye el elemento natural y fundamental de la sociedad y tiene derecho a la protección de la sociedad y del Estado.

Al nacer, el niño(a) automáticamente se incorpora a la clase social y a la posición étnica de sus padres, nace con lo que conocemos como "personalidad jurídica", que no es sino la disposición para ser facultativo de relaciones legales y cualquiera que pueda ser su futuro, la criatura no tiene una posición individual sino que recibe la que su familia le inculca, por ejemplo, cuando la madre elige a los compañeros de juego de sus hijo, le está dando su identificación de clase con otros niños, y de acuerdo a lo establecido en la Constitución de la República del Ecuador (2008):

Artículo 66.- Se reconoce y garantizará a las personas: numeral 28: El derecho a la identidad personal y colectiva, que incluye tener nombre y apellido, debidamente registrados y libremente escogidos; y conservar, desarrollar y fortalecer las características materiales e inmateriales de la identidad, tales como la nacionalidad, la procedencia familiar, las manifestaciones espirituales, culturales, religiosas, lingüísticas, políticas y sociales.

En la legislación ecuatoriana no existe un cuerpo independiente de normas que regulen todo lo referente a la familia, pero, partiendo desde la Constitución de la República del Ecuador (2008), sin dejar de considerar otras normas de carácter secundario, encontramos disposiciones que tratan de proteger a la familia como Institución, en el 
Título II denominado "Derechos", Capítulo VI "Derechos de la Libertad", establece:

Artículo. 67.- Se reconoce a la familia en sus diversos tipos. El Estado la protegerá como núcleo fundamental de la sociedad y garantizarácondiciones que favorezcan integralmente la consecución de sus fines. Estas se constituirán por vínculos jurídicos o de hecho y se basarán en la igualdad de derechos y oportunidad de sus integrantes. El matrimonio es la unión entre hombre y mujer, se fundará en el libre consentimiento de las personas contrayentes y en la igualdad de sus derechos, obligaciones y capacidad legal.

Es por ello que, al referirnos a la familia, en primera instancia, debemos reconocer, no sólo su importancia, su evolución, su desarrollo, sino la influencia que ha tenido a lo largo de la historia y que ha conllevado a que los Estados tengan la obligación de protegerla.

De acuerdo a Solano-Paucay \& Verdugo-Silva (2021) la Corte Constitucional Ecuatoriana ha generado sentencias en el orden de preservar "el derecho de identidad, filiación y tenencia, de los menores, lo cual genera jurisprudencia para establecer sentencias en el orden de preservar derechos como alimentación, vivienda, educación, entre otros, para establecer el principio de crecimiento integral de la persona". (p. 18)

En su autoanálisis el hombre ha podido apreciar la necesidad de vivir en colectividad, en grupo, ha constituido una de las bases que ha dado origen a la familia considerada como una institución social, cuyos miembros, no solo que forman parte de la misma y se hallan unidos sea por vínculos consanguíneos, afinidad, adopción, por afectos, sino que, además, mantienen derechos y obligaciones.

Los derechos de la familia, está organizado por el conjunto de normas, principios y pautas que constituyen parte del Derecho Privado; así, por ejemplo, encontramos en nuestra legislación: normas referentes a la familia en la Constitución de la República, además, en leyes secundarias como el Código Civil, el derecho patrimonial, el Código Orgánico de la Niñez y la Adolescencia (CONA) el cual contiene un conjunto de disposiciones que no solo están llamadas a garantizar los derechos de los niños, niñas y adolescentes, sino que, igualmente, encontramos aquellas correspondientes con los progenitores en relación hacia sus hijos, y las garantías para los niños, niñas y adolescentes a conservar 
la familia, así, el CONA en su Artículo 22 establece el derecho a poseer una familia y a la convivencia dentro de la misma. Excepcionalmente, cuando aquello sea imposible o contradictorio a su interés superior, los niños, niñas y adolescentes poseen derecho a una nueva familia, de consentimiento con la ley.

En este sentido la Declaración Universal de los Derechos Humanos, establece:

Artículo 16. 1. Los hombres y las mujeres, a partir de la edad núbil, tienen derecho, sin restricción alguna por motivos de raza, nacionalidad o religión, a casarse y fundar una familia; y disfrutarán de iguales derechos en cuanto al matrimonio, durante el matrimonio y en caso de disolución del matrimonio. 2. Sólo mediante libre y pleno consentimiento de los futuros esposos podrá contraerse el matrimonio. 3. La familia es el elemento natural y fundamental de la sociedad y tiene derecho a la protección de la sociedad y del Estado

Es importante enfatizar que ninguna otra rama del Derecho tiene tan íntima relación con la moral como el derecho de la familia. Las reglas éticas lo han marcado notablemente, reflejando en nuestra cultura la influencia de la Religión Católica y concretamente del Derecho Canónico que triunfó en la organización familiar e imperó en nuestro país y en gran parte del mundo occidental e inspiró las principales instituciones a pesar de la laicización del matrimonio.

En tal sentido, el funcionamiento físico, social y emocional de los integrantes de la familia es el resultado del interactuar y consecuencias no solo que afectan al interior de la misma, sino que puede generar impactos en la sociedad, cualquier modificación en un fragmento del sistema, resuena en otras porciones del mismo, además las interacciones y relaciones familiares tienden a ser altamente recíprocas, modeladas y repetitivas, es esta redundancia de patrones la que nos permite hacer predicciones, obtener información, llegar a conclusiones del porqué del actuar de uno u otro miembro de la familia. Al respecto Alarcón-Cedeño \& Suárez-Montes (2020), la define como:

La familia es una institución inserta en todas las sociedades humanas, adicionalmente reconocida entre los factores más valorados en la vida. Porlo tanto, conforma parte del mecanismo natural, así como elemental, de la 
sociedad, en consecuencia, merece y debe ser protegida por el Estado, a través del institucionalismo jurídico y basamento legal. (p.1023).

Desde otra perspectiva, el conjunto familiar como sistema, posee limitaciones que componen su ámbito, estos tienen el propósito de mantener a sus miembros, resguardarlo de las amenazas externas y vigilar el intercambio de información que ingresa y emerge en sus relaciones y contacto con el contexto, de modo que desempeñan situaciones tantas defensoras a modo reguladora, con el objeto de preservar a sus miembros en unidad y al sistema constante, cuando la impregnación de los limites es enorme, el sistema logra disipar la identificación e integridad, y, cuando es insuficiente el sistema se obstruye y se incomunica.

Brindar un concepto definitivo sobre la familia es una acción complicada en virtud de la gran diversidad que de ella descubrimos y que se han distinguido en enunciados anteriores. Para Fuente-Linares (2012), la familia es:

Aceptar que la familia es un grupo social, que es la célula de toda sociedad, que es un grupo natural, que es un grupo primario, se le debe regular como tal, como grupo, atendiendo al interés superior de la familia constituida por todos sus elementos, no podemos enfrentar los intereses de cada uno: del niño, la niña, el adolescente, el joven, el de la tercera edad, la mujer, como si fueran individualidades, tenemos que compatibilizar los intereses de todos para poder lograr el interés de la unidad del todo; el Estado es lo que son sus familias, la humanidad es lo que son sus Estados, tenemos que partir por el concepto de esa base y regular a la familia como núcleo o célula de la sociedad, integrada por distintos individuos unidos de manera indisoluble, de manera inseparable, sólo así puedo entender a la familia.(p.63)

Al hablar del parentesco, hay que considerar lo relacionado a la línea y el grado, formas o mecanismos de calcular, de establecer, de ubicar el parentesco entre las personas. El parentesco en línea permite establecer la cadena de parentelas que provienen unos de los otros o de un tronco común, puede ser recta, colateral, transversal u oblicua. Finalmente, el parentesco en línea recta se constituye cuando los parientes descienden uno del otro: padre e hijo, abuelo - nieto. El parentesco en línea recta se cuenta por la cantidad de procreaciones, así el hijo está en el primer grado de consanguinidad en línea recta con sus padres, porque del padre al hijo hay una sola generación: la del hijo. Por 
eso se dice es el descendiente en primer grado. Al respecto el artículo 22 del Código Civil Ecuatoriano (2015), establece en relación a la consanguineidad lo siguiente:

Los grados de consanguinidad entre dos personas se cuentan por el número de generaciones. Así, el nieto está en segundo grado de consanguinidad con el abuelo; y los primos hermanos, en cuarto grado de consanguinidad entre sí. Cuando una de las dos personas es ascendiente de la otra, la consanguinidad es en línea recta; y cuando las dos personas proceden de un ascendiente común, y una de ellas no es ascendiente de la otra, la consanguinidad es en línea colateral o transversal...

Respecto al grado, estas son las distancias o espacios existentes entre dos parientes, tiene importancia determinarlo porque mientras más cercano es el grado de parentesco, mayores son los derechos que él origina. Así, por ejemplo, los hermanos son parientes en segundo grado de consanguinidad en línea colateral, porque de un hermano subimos al padre un grado y de este bajamos al otro hermano otro grado. Por lo que el vínculo más cercano en la línea colateral es el de segundo grado, es decir, los hermanos, pues en esta línea colateral no hay parientes en primer grado. La importancia del parentesco conforme se ha señalado radica en que entre más cercano sea el grado parental mayores son los derechos como las obligaciones, así tenemos por ejemplo que de conformidad a lo que contempla el artículo innumerado 5 de la Ley Reformatoria al Código de la Niñez y Adolescencia (2017):

Obligados a la prestación de alimentos. - Los padres son los titulares principales de la obligación alimentaria, aún en los casos de limitación, suspensión o privación de la patria potestad. En caso de: ausencia, impedimento, insuficiencia de recursos o discapacidad de los obligados principales, debidamente comprobado por quien lo alega, la autoridad competente ordenará que la prestación de alimentos sea pagada 0 completada por uno o más de los siguientes obligados subsidiarios, en atención a su capacidad económica y siempre y cuando no se encuentren discapacitados, en su orden: 1. Los abuelos/as; 2. Los hermanos/as que hayan cumplido 21 años y no estén comprendidos en los casos de los numerales dos y tres del artículo anterior; y, 3. Los tíos/as. 
La norma legal indica que son los miembros de la familia consanguínea los primeros en responder, en asumir la obligación connatural denominada pensión alimenticia. Podemos concluir que tres son los efectos principales del parentesco y que se manifiestan en los derechos, las obligaciones y las incapacidades, o, para hablar con mayor propiedad, las prohibiciones que se originan del parentesco. Los derechos que nacen del parentesco guardan relación con el matrimonio, la patria potestad y los alimentos que se deben por Ley a ciertas personas; con la constitución del patrimonio familiar y el derecho de usufructo legal, con los órdenes de la sucesión intestada y las llamadas asignaciones forzosas y, en fin, con la representación.

Por lo tanto, la familia es la más compleja de todas las instituciones, aunque en nuestra sociedad muchas de sus actividades tradicionales hayan pasado parcialmente a otras, todavía quedan sociedades en las que la familia continúa ejerciendo las funciones educativas, religiosas, protectoras, recreativas y productivas. En ocasiones algunos padres transfieren a otras instituciones las tareas familiares, no porque la familia sea incapaz de cumplir con su deber, sino porque las actividades que realizan en la actualidad requieren del apoyo de otros organismos que les proporcionen un medio eficaz de conseguir los mismos propósitos. Entre las más importantes se señala a la escuela, pues los padres por la comodidad, trabajo, falta de tiempo dejan la formación, educación y el ejemplo a seguir en manos de terceros.

Indudablemente a través de la historia y sin lugar a discusión, la familia resulta ser la más antigua y permanente de las instituciones sociales. Varios criterios, tesis, comentarios y discusiones se han propiciado sobre las primeras familias, cuando el hombre ya tuvo su condición de tal, diferenciándose claramente de los demás primates. La observación de las sociedades humanas primitivas dio como resultado conocer que aquéllas tenían un ordenamiento sexual basado en el instinto procreador. El número de esposos era circunstancial basado en el excedente de mujeres, que era el caso más común, rara vez ocurría lo contrario, practicándose indistintamente la poligamia o la poliandria de acuerdo a las circunstancias. Pero, además de las motivaciones sexuales, en los seres humanos 
los instintos sociales son congénitos al hombre normal, para quien es un castigo vivir en la soledad, lo que demuestra desde niño. Hay autores que sostienen que el instinto familiar se desarrolla primero en los varones, siendo este criterio discutible, pues el instinto maternal de la mujer estaba más desarrollado que el paternal del hombre, siendo talvez una de las explicaciones por las cuales se da origen a la familia.

Siendo la familia, la fuente de la vida, es el principal vínculo vital y orgánico de la sociedad, los integrantes de la sociedad son inicialmente integrantes de la familia y allí reciben el primer aprendizaje de las virtudes sociales, que luego se transformarán en virtudes cívicas. La familia cumple una función social al relacionarse con otras familias y dando lugar desde tiempos inmemoriales a la formación de sociedades cada vez mayores. La familia es la principal escuela de civilidad y el estímulo para las demás relaciones comunitarias. Para Morales (2015):

El concepto jurídico de familia sólo se considera a partir, de la pareja, sus descendientes y ascendientes, la importancia de la familia radica en que se constituye en la primera institución socializadora y generadora de relaciones de poder, que se detenta en la mayoría de las veces por la aportación económica que uno de sus miembros realiza en mayor proporción. (p.149)

Además de la función procreadora y educativa, la familia tiene otras funciones que cumplir. Debe tener una directa intervención en los asuntos del Estado, como es el control de las Leyes, la solución de las dificultades que inquietan a toda la sociedad, la participación en las acciones encaminadas a terminar con la injusticia social existente. Esta participación la hace a través de sus miembros adultos, en el momento actual no puede mantenerse al margen del desenvolvimiento histórico.

Por todo lo planteado, la investigación tiene como objetivo general analizar desde la perspectiva jurídica la visión de la familia en Ecuador. 


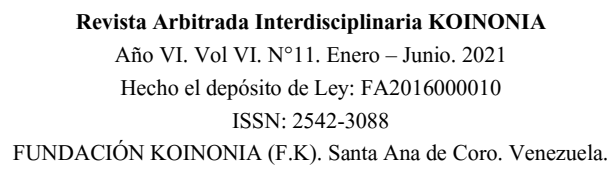

Nancy Susana Cárdenas-Yánez; Vicente Manuel Solano-Paucay; Lourdes Álvarez-Coronel; María Elena Coello-Guerrero

\section{METODOLOGÍA}

La investigación se llevó a cabo desde el método analítico - sintético y se desarrolló desde un tipo descriptivo documental, empleando la observación y análisis de contenido jurídico con el propósito de descubrir las ideas primordiales de leyes, dictámenes, trabajos de investigación, conexas con el tema, lo cual accedió construir un sumario teórico del caso de estudio, los mismos se presentan en los resultados investigativos. En tal sentido Hoyos-Botero, citado por Guevara Patiño (2016) plantea lo siguiente:

El estado del arte es una investigación documental que tiene un desarrollo propio, cuya finalidad esencial es dar cuenta de construcciones de sentido sobre bases de datos que apoyan un diagnóstico y un pronóstico en relación con el material documental sometido a análisis. Implica, además, una metodología mediante la cual se procede progresivamente por fases bien diferenciadas para el logro de unos objetivos delimitados que guardan relación con el resultado del proceso. (p.169)

\section{RESULTADOS Y DISCUSIÓN}

En el siguiente a apartado se muestra el resultado en el orden jurídico y normativo en cuanto a la perspectiva familiar.

\section{Tabla 1.}

Preceptos constitucionales.

\begin{tabular}{|l|l|}
\hline \multicolumn{1}{|c|}{ Normativa } & \multicolumn{1}{c|}{ Contenido } \\
\hline $\begin{array}{l}\text { Constitución de la } \\
\text { República del Ecuador } \\
\text { Articulo 44 }\end{array}$ & $\begin{array}{l}\text { Sección quinta } \\
\text { Niñas, niños y adolescentes El Estado, la sociedad y la familia } \\
\text { promoverán de forma prioritaria el desarrollo integral de las } \\
\text { niñas, niños y adolescentes, y asegurarán el ejercicio pleno de } \\
\text { sus derechos; se atenderá al principio de su interés superior y sus } \\
\text { derechos prevalecerán sobre los de las demás personas. }\end{array}$ \\
\hline Artículo 47 & $\begin{array}{l}\text { Sección sexta } \\
\text { Personas con discapacidad. - El Estado garantizará políticas de } \\
\text { prevención de las discapacidades y, de manera conjunta con la } \\
\text { sociedad y la familia, procurará la equiparación de } \\
\text { oportunidades para las personas con discapacidad y su } \\
\text { integración social. }\end{array}$ \\
\hline Artículo 49 & $\begin{array}{l}\text { Las personas y las familias que cuiden a personas con } \\
\text { discapacidad que requieran atención permanente serán cubiertas }\end{array}$ \\
\hline
\end{tabular}




\begin{tabular}{|c|c|}
\hline & $\begin{array}{l}\text { por la Seguridad Social y recibirán capacitación periódica para } \\
\text { mejorar la calidad de la atención }\end{array}$ \\
\hline Artículo 67 & $\begin{array}{l}\text { Se reconoce la familia en sus diversos tipos. El Estado la } \\
\text { protegerá como núcleo fundamental de la sociedad y } \\
\text { garantizará condiciones que favorezcan integralmente la } \\
\text { consecución de sus fines. Estas se constituirán por vínculos } \\
\text { jurídicos o de hecho y se basarán en la igualdad de derechos y } \\
\text { oportunidades de sus integrantes. } \\
\text { El matrimonio es la unión entre hombre y mujer, se fundará en el } \\
\text { libre consentimiento de las personas contrayentes y en la } \\
\text { igualdad de sus derechos, obligaciones y capacidad legal. }\end{array}$ \\
\hline Artículo 68 & $\begin{array}{l}\text { La unión estable y monogámica entre dos personas libres de } \\
\text { vínculo matrimonial que formen un hogar de hecho, por el lapso y } \\
\text { bajo las condiciones y circunstancias que señale la ley, generará } \\
\text { los mismos derechos y obligaciones que tienen las familias } \\
\text { constituidas mediante matrimonio. } \\
\text { La adopción corresponderá sólo a parejas de distinto sexo. }\end{array}$ \\
\hline Artículo 69 & $\begin{array}{l}\text { Para proteger los derechos de las personas integrantes de la } \\
\text { familia: } \\
\text { 1. Se promoverá la maternidad y paternidad responsables; la } \\
\text { madre y el padre estarán obligados al cuidado, crianza, } \\
\text { educación, alimentación, desarrollo integral y protección de los } \\
\text { derechos de sus hijas e hijos, en particular cuando se encuentren } \\
\text { separados de ellos por cualquier motivo. } \\
\text { 2. Se reconoce el patrimonio familiar inembargable en la cuantía } \\
\text { y con las condiciones y limitaciones que establezca la ley. Se } \\
\text { garantizará el derecho de testar y de heredar. } \\
\text { 3. El Estado garantizará la igualdad de derechos en la toma de } \\
\text { decisiones para la administración de la sociedad conyugal y de la } \\
\text { sociedad de bienes. } \\
\text { 4. El Estado protegerá a las madres, a los padres y a quienes } \\
\text { sean jefas y jefes de familia, en el ejercicio de sus obligaciones, } \\
\text { y prestará especial atención a las familias disgregadas por } \\
\text { cualquier causa. } \\
5 \text {. El Estado promoverá la corresponsabilidad materna y paterna } \\
\text { y vigilará el cumplimiento de los deberes y derechos recíprocos } \\
\text { entre madres, padres, hijas e hijos. } \\
6 \text {. Las hijas e hijos tendrán los mismos derechos sin considerar } \\
\text { antecedentes de filiación o adopción. } \\
\text { 7. No se exigirá declaración sobre la calidad de la filiación en el } \\
\text { momento de la inscripción del nacimiento, y ningún documento de } \\
\text { identidad hará referencia a ella. }\end{array}$ \\
\hline
\end{tabular}


Los apartados referidos, se centran en la protección del bienestar familiar con el propósito de la promoción desde la constitución la importancia para la sociedad, su protección integral para plasmar y cumplir con el buen vivir desde un enfoque jurídico y sociológico donde la familia se compone en el centro de las políticas del Estado, en tan sentido la familia se divisa como la base elemental.

\section{Tabla 2.}

Ley vinculante.

\begin{tabular}{|c|c|}
\hline Normativa & Contenido \\
\hline $\begin{array}{l}\text { LEY CONTRA LA VIOLENCIA A LA } \\
\text { MUJER Y A LA FAMILIA } \\
\text { Artículo } 1\end{array}$ & $\begin{array}{l}\text { TÍTULO PRELIMINAR } \\
\text { FINES DE LA LEY. - la presente Ley tiene por } \\
\text { objeto proteger la integridad física, psíquica y } \\
\text { libertad sexual de la mujer y los miembros } \\
\text { de su familia, mediante la prevención y } \\
\text { sanción de la violencia intrafamiliar y los } \\
\text { demás atentados contra sus derechos y los de } \\
\text { su familia. Sus normas deben orientar las } \\
\text { políticas del Estado y la comunidad sobre la } \\
\text { materia. }\end{array}$ \\
\hline Artículo 2 & $\begin{array}{l}\text { VIOLENCIA INTRAFAMILIAR. Se considera } \\
\text { violencia intrafamiliar toda acción u omisión } \\
\text { que consista en maltrato físico psicológico o } \\
\text { sexual ejecutado por un miembro de la } \\
\text { familia en contra de la mujer o demás } \\
\text { integrantes del núcleo familiar. }\end{array}$ \\
\hline Artículo 3 & $\begin{array}{l}\text { AMBITO DE APLICACION. - para los efectos } \\
\text { de esta ley se consideran miembros del } \\
\text { núcleo familiar a los cónyuges, } \\
\text { ascendientes, descendientes, hermanos y sus } \\
\text { parientes hasta el segundo grado de afinidad. } \\
\text { La protección de esta Ley se hará extensiva a } \\
\text { los excónyuges, convivientes, ex- } \\
\text { convivientes, a las personas con quienes se } \\
\text { mantenga o se haya mantenido una relación } \\
\text { consensual de pareja, así como a quienes } \\
\text { comparten el hogar del agresor o del agredido. }\end{array}$ \\
\hline Artículo 4 & $\begin{array}{l}\text { FORMAS DE VIOLENCIA INTRAFAMILIAR. - } \\
\text { Para los efectos de esta Ley, se considera: } \\
\text { Art. 4.- FORMAS DE VIOLENCIA } \\
\text { INTRAFAMILIAR. - Para los efectos de esta } \\
\text { Ley, se considera: }\end{array}$ \\
\hline
\end{tabular}


Elaboración propia (2021).

Se establecen leyes que vincular la vida familiar y otros hechos que vulneran mediante hechos de violencia la tranquilidad del desarrollo saludable intrafamiliar.

En otro cuerpo legar como lo es el Código Civil se establecen derechos y obligaciones en los cuales se involucra la familia, lo cual condiciona la relación y desarrollo desde el punto de vista jurídico desde lo consanguíneo, la protección, las imposiciones dinerarias, entre otros aspectos que en un momento dado vulneran el buen desarrollo intrafamiliar.

\section{CONCLUSIONES}

La Familia, cualquiera sea su organización, constituye una necesidad inevitable para el desenvolvimiento de las personas en la colectividad. En las sociedades primitivas el origen de la Familia era matriarcal, luego de la etapa de promiscuidad sexual y de un significativo cambio socio económico decae el Matriarcado para dar paso al Patriarcado. En la Edad Media la familia constituyó una verdadera organización económica caracterizada por su autoabastecimiento, progresivamente el sistema se modifica y la economía se traslada a los mercados, corporaciones, organizaciones capitalistas y al Estado.

En los puntos de análisis se puede inferir que una de las características fundamentales de la familia contemporánea se refleja en el sistema monogámico, inclusive, la unión de hecho para ser protegida por el Estado debe ser firme y monogámica entre dos personas libres de lazo matrimonial. El Estado reconoce a la Familia como el núcleo fundamental de la sociedad, en sus diversos tipos, obligándose a garantizar las condiciones necesarias para el acatamiento de sus fines. La Familia está tutelada por normas de disposición pública, no logran desconocerse, así como tampoco es posible anteponer el interés individual por sobre el interés de la sociedad. El Derecho de Familia está 
establecido por un conjunto de principios y normas de orden personal y patrimonial indispensables para su ordenación, funcionamiento, desarrollo, cumplimiento de sus fines y disolución.

Existe, en esta institución, la Familia, una marcada influencia principalmente de la moral, así como también de la religión y las costumbres. La Familia es preocupación fundamental del Derecho, éste la regula desde sus diferentes ámbitos: público, privado y social. La familia determina el goce de ciertos beneficios y el cumplimiento de obligaciones que tiene el trabajador, por lo tanto, es una institución importante en el desarrollo del Derecho del Trabajo, que igualmente se viene ajustando a las nuevas modalidades y estructuras familiares.

\section{FINANCIAMIENTO}

No monetario.

\section{AGRADECIMIENTO}

A la Universidad de Cuenca; por motivar el desarrollo de la investigación.

\section{REFERENCIAS CONSULTADAS}

Alarcón-Cedeño, F., \& Suárez-Montes, N. (2020). La familia como eje transformador de la sociedad sustentada en el ámbito jurídico. [The family as the transforming axis of society sustained in the legal field]. Polo del Conocimiento. 5(10), 1011-1026. DOI: $\underline{10.23857 / p c . ~ v 5 i 10.2140}$

Asamblea Nacional (2009). Ley Reformatoria al Código de la Niñez y Adolescencia. [Law Reforming the Code of Children and Adolescents]. Recuperado de: https://n9.cl/fbcka

Benítez-Pérez, M. (2017). La familia: Desde lo tradicional a lo discutible. [The family: From the traditional to the debatable]. Revista Novedades en Población, 13(26), 58-68. Recuperado de: https://n9.cl/2xxn

Congreso Nacional (2015). Código Civil del Ecuador. [Ecuador Civil Code] Registro Oficial Suplemento 46 de 24-jun.-2005 Ultima modificación: 19-jun.-2015 Estado: Vigente. Recuperado de: https://n9.cl/she7c 
Congreso Nacional (2003). Código de la Niñez y Adolescencia. [Code of Childhood and Adolescents]. publicado por Ley No. 100. en Registro Oficial 737 de 3 de enero del 2003. Recuperado de: https://n9.cl/dtrec

Congreso Nacional (1995). Ley Contra la Violencia a la Mujer y la familia. [Law Against Violence against Women and the Family]. Registro Oficial No 839 del 11 de diciembre 1995. Recuperado de: https://n9.cl/vy46s

Constitución de la República del Ecuador (2008). [Constitution of the Republic of Ecuador]. Asamblea Nacional Constituyente. Montecristi, Manabí, Ecuador: Registro Oficial 449 de 20-oct-2008

Fuente-Linares, José Cándido Francisco Javier de la. (2012). La protección constitucional de la familia en América Latina. [The constitutional protection of the family in Latin America]. Revista IUS, 6(29), 60-76. Recuperado de https://n9.cl/eqlt

Guevara-Patiño, R. (2016). El estado del arte en la investigación: ¿análisis de los conocimientos acumulados o indagación por nuevos sentidos? [The state of the art in research: analysis of accumulated knowledge or inquiry into new senses?]. FOLIOS, 2(44), 165-179. Recuperado de: http://www.scielo.org.co/pdf/folios/n44/n44a11.pdf

Morales-Gómez, S. (2015). La familia y su evolución. [The family and its evolution]. Perfiles De Las Ciencias Sociales, 3(5),128-155. Recuperado de: https://revistas.ujat.mx/index.php/perfiles/article/view/1038

Naciones Unidas (1948). Declaración Universal de Derechos Humanos. [Universal Declaration of Human Rights]. Recuperado de https://url2.cl/d9qxa

Oliva-Gómez, E., \& Villa-Guardiola, V. (2014). Hacia un concepto interdisciplinario de la familia en la globalización. [Towards an interdisciplinary concept of family in Globalization]. Justicia Juris, 10(1), 11-20. Recuperado de: http://www.scielo.org.co/pdf/jusju/v10n1/v10n1a02.pdf

Constitución de la República del Ecuador (2008). [Constitution of the Republic of Ecuador]. Asamblea Nacional Constituyente. Montecristi, Manabí, Ecuador: Registro Oficial 449 de 20-oct-2008 


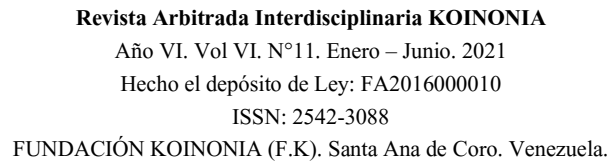

Nancy Susana Cárdenas-Yánez; Vicente Manuel Solano-Paucay; Lourdes Álvarez-Coronel; María Elena Coello-Guerrero

Solano-Paucay, V., \& Verdugo-silva, J. (2021). Jurisprudencia de la Corte Constitucional del Ecuador en protección de los derechos de los menores. [Jurisprudence of the Constitutional Court of Ecuador in protection of the rights of minors]. Iustitia Socialis, 6(10), 4-21. http://dx.doi.org/10.35381/racii.v6i10.1125

(C2021 por los autores. Este artículo es de acceso abierto y distribuido según los términos y condiciones de la licencia Creative Commons Atribución-NoComercial-Compartirlgual 4.0 Internacional (CC BY-NC-SA 4.0)

(https://creativecommons.org/licenses/by-nc-sa/4.0/). 